\title{
Research Article \\ Energy Dependent Divisible Load Theory for Wireless Sensor Network Workload Allocation
}

\author{
Haiyan Shi, ${ }^{1,2}$ Wanliang Wang, ${ }^{1}$ and Ngaiming Kwok ${ }^{3}$ \\ ${ }^{1}$ College of Computer Science, Zhejiang University of Technology, Hangzhou 310023, China \\ ${ }^{2}$ School of Computer Science and Technology, Shaoxing University, Shaoxing 312000, China \\ ${ }^{3}$ School of Mechanical and Manufacturing Engineering, The University of New South Wales, \\ Sydney 2052, Australia
}

Correspondence should be addressed to Haiyan Shi, shi_haiyan@163.com

Received 31 October 2012; Accepted 4 December 2012

Academic Editor: Sheng-yong Chen

Copyright (C) 2012 Haiyan Shi et al. This is an open access article distributed under the Creative Commons Attribution License, which permits unrestricted use, distribution, and reproduction in any medium, provided the original work is properly cited.

\begin{abstract}
The wireless sensor network (WSN), consisting of a large number of microsensors with wireless communication abilities, has become an indispensable tool for use in monitoring and surveillance applications. Despite its advantages in deployment flexibility and fault tolerance, the WSN is vulnerable to failures due to the depletion of limited onboard battery energy. A major portion of energy consumption is caused by the transmission of sensed results to the master processor. The amount of energy used, in fact, is related to both the duration of sensing and data transmission. Hence, in order to extend the operation lifespan of the WSN, a proper allocation of sensing workload among the sensors is necessary. An assignment scheme is here formulated on the basis of the divisible load theory, namely, the energy dependent divisible load theory (EDDLT) for sensing workload allocations. In particular, the amount of residual energies onboard sensors are considered while deciding the workload assigned to each sensor. Sensors with smaller amount of residual energy are assigned lighter workloads, thus, allowing for a reduced energy consumption and the sensor lifespan is extended. Simulation studies are conducted and results have illustrated the effectiveness of the proposed workload allocation method.
\end{abstract}

\section{Introduction}

Sensors are devices that are frequently used in surveillance applications including industrial plant conditions, highway traffics, building security, and environment monitoring. When sensors are wire connected, their deployments or relocations may be severely hindered [13]. With advancements in sensing, electronics, and radio technologies, sensors equipped with computation and communication capabilities have become available. A wireless sensor network (WSN) can then be formed to perform an assigned surveillance task independently 
[4] or in a collaborative manner [5]. Usually, each sensor in a WSN collects sensing data and then sends the result to a central processor for further analysis. In order to maximize the data transfer efficiency, careful transmission routing designs are required [6]. On the other hand, wireless sensors are embedded with only onboard batteries as the sole power source. Because the deployment of wireless sensors is mostly carried out in an ad hoc manner, it may be difficult to gain access to their locations and makes battery replacements difficult. Hence, it is very desirable that the rate of battery energy consumption could be carefully managed [7]. Otherwise, some form of energy harvesting [8] is needed to ensure that the sensor can operate in an extended duration. Such approach, however, is complicated and may increase the cost in setting up the WSN. Alternatively, approaches to prolong the operation duration by choosing data transmission routes [9] can be properly determined to reduce battery energy consumption.

In addition to properly determine the data transmission route, it is also possible to reduce sensor energy consumption by dividing the sensing workload appropriately among elements in the WSN [10]. This approach is frequently developed by making use of the divisible load theory (DLT) [11]. In the theory, it is assumed that the computation or sensing workload can be arbitrarily divided into portions [12] so that the time to complete the task could be minimized. The DLT has been applied in computation load sharing among distributed processors [13] because of its tractability in closed-form solutions. This theory is also generic enough that it has been used in load scheduling for a variety of network architectures such as systems having time-varying computation speeds [14]. The DLT was employed in a bus network of processors [15] while the cost of computation was optimized. Furthermore, DLT can also be applied in the scheduling of loads on a star and tree network [16] where the theory was used to assign loads to processors in multirounds.

For wireless sensor networks, scheduling is concerned with proper assignments of the sensing and result reporting workloads [17] corresponding to the computation and communication phases in distributed networks. For example, in [18], several sensing and reporting strategies were studied and their performances were evaluated by the use of DLT. These strategies include simultaneous sensing start and sequential report, simultaneous sensing start and simultaneous report termination, concurrent sensing, and reporting. Each strategy is adopted on the basis of the network architecture whether coprocessors or multichannel communication is available. Although the DLT can be applied for wireless sensor network workload scheduling, the operation of a WSN is quite different from that of computer networks. Specifically, sensors engaged in the WSN operate only from onboard battery energies and the amount of such energy is always limited.

Energy consumption is a prominent issue that network designers have to take into consideration [19] even for distributed computer networks. Because of the need for WSNs to transmit the sensed data to a master processor, a majority of energy would be consumed therein. Therefore, in order to mitigate the adverse effect of energy depletion on network lifetime, sensors are formed into clusters depending on their physical location vicinity [20]. Clustering itself is a challenging task for deterministic approaches that designer may employ soft computing techniques to design clusters [21-27]. A further development, the low-energy adaptive clustering hierarchy $(\mathrm{LEACH})$ protocol was reported in [28] that sensor clusters are formed according to the sensor locations with the objective to minimize the overall energy consumed by the sensors in the network. An alternative scheme was presented [29] where the sensor network lifetime was extended by dynamically adjusting the transmission routes. Furthermore, the minimization of total energy consumed along a sensor data reporting route was considered [30] for multihop transmission. Moreover, an attempt was made to employ 
a spanning tree [31] representation of the sensed data reporting route which resulted in the reduction of sensor energy consumption.

Issues on sensor energy consumption are receiving a considerable amount of recent research attention. Its reduction to prolong network lifetime has also been tackled from diversified perspectives. For example, sensor clusters were formed and the cluster head, responsible to process or retransmit the sensed data, was elected in accordance with the ratio of residual energies of neighboring sensors [32]. By building on the cluster structure, hierarchies of sensor clusters were constructed to carry out a sensing task in a coordinated manner [33] in order to minimize the energy used in radio transmission. Moreover, a challenging design case [34], where network communication resources such as sensing and transmission speed were not known, was approached using an adaptive strategy to allocate sensing workloads.

In the reported works, attempts to extend network lifetime were made by using the proper workload allocation strategy or the transmission routing optimization strategy in order to minimize energy consumption. However, it has not been studied in detail how workload division can effectively increase the WSN operation duration. Here, the divisible load theory is adopted for WSN workload assignment. The modification on DLT makes use of the ratio of the initial to residual sensor energies when the workload allocated to an individual sensor is determined. By adopting this design philosophy, sensors having a lesser amount of remaining energy will be given a smaller portion of sensing workload and provides a reduction on its energy consumption. The overall result is that the energy depletion time of each sensor is prolonged; hence, the whole wireless sensor network can operate for an extended duration.

The rest of the paper is organized as follows. In Section 2, the WSN structure is firstly presented and the application of DLT is described. In Section 3, the energy consumption profile is formulated and the development of the proposed energy dependent divisible load theory is given. Simulations to verify the proposed method are described and results are presented in Section 4. Finally, a conclusion is drawn in Section 5.

\section{Wireless Sensor Network Workload Allocation}

Sensors in the network consume battery energy in their electronic circuits when carrying out sensing and radio transmission [35]. In practice, the amount of energy used is proportional to the duration that these activities are being conducted. In order to reduce the rate of energy consumption, it is desirable that sensing and reporting operations can be completed in the shortest time. It is also assumed that, in terms of some demanded sensing precision that leads to a certain quantity of measured data required, the sensing workload can be divided among sensors in the network. To this end, the divisible load theory is applied to derive a set of optimal workloads in the sense of minimum sensing completion time and minimum sensor energy consumption. In the following, the WSN architecture adopted in the current work is presented and the enhancement of DLT in determining workloads is illustrated.

\subsection{Wireless Sensor Network Architecture}

Let the wireless sensor network contains $N$ sensors clustered in a star topology [16], see Figure 1. Sensors $S_{i}, i=1, \ldots, N$, are responsible to measure the environment and report 


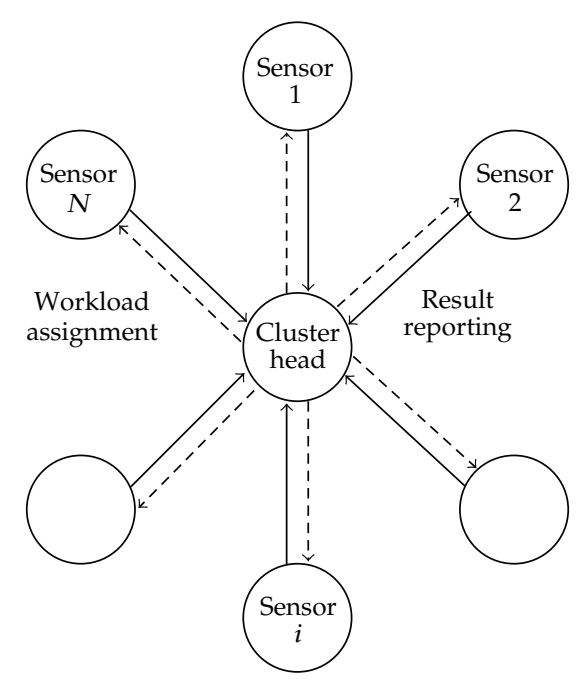

Figure 1: Star network architecture.

the results via wireless communication to the cluster head $\mathrm{CH}$. The cluster head relays workload assignments from the master processor to sensors, performs data aggregation and transmits them back to the master processor for analysis [18]. On the other hand, the $\mathrm{CH}$ does not take measurements nor reports its own data. It is also assumed that the network operates on a single two-way channel but radio communication is only permitted for onedirection transmission from a sole originator at any time. Thus, workload assignments are transmitted from the $\mathrm{CH}$ to sensors sequentially. Furthermore, sensors report the sensed results to the $\mathrm{CH}$ via the same radio channel also in the sequential transmission mode. Each sensor receives the workload assignment from the $\mathrm{CH}$ but measurement is allowed to commence only after the last sensor has received its workload allocation command. The sensor then carries out sensing the environment and reports the results when the measurement is completed. The time and amount of result data to be sent to the $\mathrm{CH}$ is proportional to the workload assigned. Moreover, it is assumed that the duration of reporting is a scaled portion of the sensing time. All sensor characteristics including energy consumption rate, sensing, and reporting rates all remain constant during the course of a sensing task. However, the residual battery onboard the sensor diminishes in proportional to the amount of sensing workload carried out as well as the distance between the $\mathrm{CH}$ and the sensor.

\subsection{Application of Divisible Load Theory}

Based on the aforementioned wireless sensor network architecture, a timing diagram could then be drawn and is depicted in Figure 2. It can be seen that the time for workload assignment $T_{a s}$ is equal for all sensors and measurement starts simultaneously for all sensors at time $T_{0}$. The sensing time for sensor $S_{i}$ is given by

$$
T_{m s, i}=\alpha_{i} y_{i} T_{m s}
$$




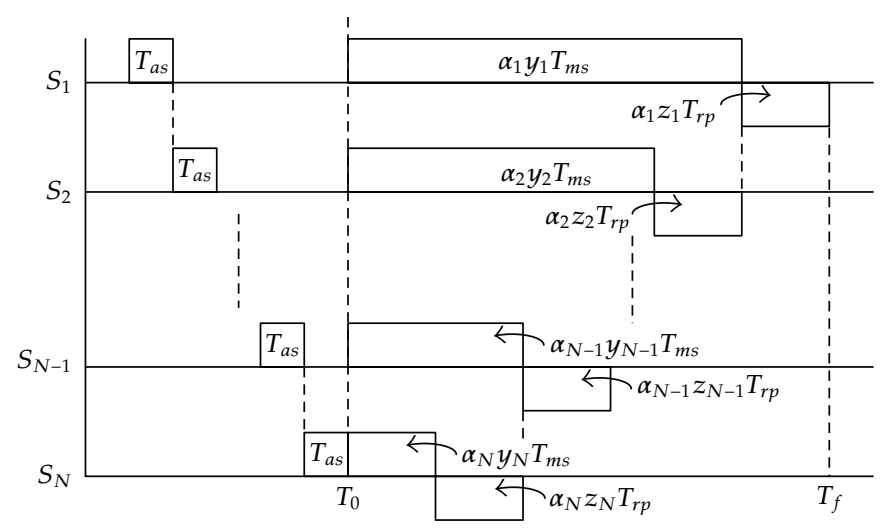

Figure 2: Sensing and result reporting schedule.

where $\alpha_{i}$ is the portion of assigned sensing workload, $y_{i}$ is a design coefficient to modify $\alpha_{i}$, and $T_{m s}$ is the sensor measurement time determined by the sensor circuitry. When sensing is completed, the sensor sends the result to the $\mathrm{CH}$ in time

$$
T_{r p, i}=\alpha_{i} z_{i} T_{r p}
$$

where $z_{i}$ is also a design parameter that modifies the radio transmission duration, $T_{r p}$ is the time used for result reporting. The overall time that sensor $S_{i}$ used for the portion of assigned workload is

$$
T_{S_{i}}=\alpha_{i} y_{i} T_{m s}+\alpha_{i} z_{i} T_{r p}
$$

Since the communication channel allows only one originator to transmit, the strategy adopted here is to align the sensing time of sensor $S_{i-1}$ to that of the overall sensing and reporting time of sensor $S_{i}$. Thus

$$
\alpha_{i-1} y_{i-1} T_{m s}=\alpha_{i} y_{i} T_{m s}+\alpha_{i} z_{i} T_{r p}
$$

This equation can be written as

$$
\alpha_{i}=s_{i} \alpha_{i-1}, \quad i=2, \ldots, N
$$

where

$$
s_{i}=\frac{y_{i-1} T_{m s}}{y_{i} T_{m s}+z_{i} T_{r p}} .
$$


The portion of workload coefficients $\alpha_{i}$ can then be obtained recursively from the following set of equations, namely,

$$
\begin{aligned}
\alpha_{2} & =s_{2} \alpha_{1} \\
\alpha_{3} & =s_{3} \alpha_{2}=s_{3} s_{2} \alpha_{1} \\
& \vdots \\
\alpha_{N} & =s_{N} \alpha_{N-1}=s_{N} s_{N-1} \cdots s_{2} \alpha_{1} .
\end{aligned}
$$

In general, we have

$$
\alpha_{i}=\alpha_{1} \prod_{j=2}^{i} s_{j} .
$$

Furthermore, when the sensing workload is normalized to unity, that is

$$
\sum_{i=1}^{N} \alpha_{i}=1
$$

then the first workload assignment $\alpha_{1}$ can be obtained from separating $\alpha_{1}$ from all other workload assignment coefficients in (2.8), then

$$
\alpha_{1}=\frac{1}{1+\sum_{i=2}^{N} \prod_{j=2}^{i} s_{j}}
$$

and the other assignments, $\alpha_{2}, \ldots, \alpha_{N}$, can be found from invoking (2.7) recursively.

\section{Energy Dependent Workload Allocation}

The assignment of proper workload to individual sensors, according to the divisible load theory, enables the measurement task to be completed in the shortest time. However, in wireless sensor networks, it is required to consider the energy consumed by each sensor in order to maintain the network in operation for an extended period. Sensors at longer distance to the cluster head may have their onboard battery energy depleted early due to the need to transmit at higher radio power. In order to compromise the task completion time and the lifespan of the whole network, workload assignments should be designed by taking the available or residual energy of sensors into consideration. First, a profile of sensor energy consumption is sketched below, then the incorporation of energy dependence into the divisible load theory is proposed. 


\subsection{Residual Energy Profile}

Consider that the master processor requires a set of $B$ bytes of data to interpret the environment being monitored. This amount of data is the quantity of report produced by all sensors and takes a total of $T_{r p}=8 \times B \tau_{b}$ seconds to complete, where $\tau_{b}$ is the time to transmit 1 bit of data. Furthermore, the time to generate the result data is directly proportional to the amount of data, that is, $T_{m s}=k T_{r p}$, where $k>0$, in general, is the scale factor representing the data compression process and depends on the sensor design. Also assume the current drained to sense a bit of data be $I$, then the energy consumed per bit in sensing is $E_{S}=V I \tau_{b}$.

Each sensor is initially installed with a battery onboard as the energy source [36]. Let the normal battery voltage be $V$ volt and the battery capacity is $A$ ampere-hour. Therefore, the initial energy carried by the sensor is

$$
\bar{E}_{0}=V A \times 60^{2} .
$$

Further assume that when the network is deployed for the first time and sensor locations are to be determined, for example, using directional antenna [37]. Throughout this localization phase, some energy $\eta_{1} \bar{E}_{0}$ would be consumed. Moreover, sensors may also form into clusters [28] and a further amount of energy $\eta_{2} \bar{E}_{0}$ is consumed. Thus, the onboard energy of a sensor before any measurement is made becomes

$$
E_{0, i}=\left(1-\eta_{1}-\eta_{2}\right) \bar{E}_{0}
$$

where $\eta_{1} \in\left[\begin{array}{ll}0.00 & 0.02\end{array}\right]$ and $\eta_{2} \in\left[\begin{array}{ll}0.00 & 0.03\end{array}\right]$ are random numbers representing the initial portion of energy usage.

For a sensor $S_{i}$ assigned with workload fraction $\alpha_{i}$, the sensing time is $T_{m s, i}=\alpha_{i} T_{m s}$. During this period, an equivalent number of data bits that consume energy are $B_{m s, i}=T_{m s, i} / \tau_{b}$ and the energy consumed is $E_{m s, i}=B_{m s, i} E_{s}$. For sensed data reporting, the report time is $T_{r p, i}=\alpha_{i} T_{r p}$, and the number of bits reported is $B_{r p, i}=T_{r p, i} / \tau_{b}$.

In addition, assume that the transmit power can be adjusted according to the distance $d$ between the sensor and the cluster head [38]. The energy consumed in transmitting the result to the cluster head is

$$
E_{r p, i}=B_{r p, i}\left(1046 \times 10^{-9}+22.2 \times 10^{-12} d^{2}\right) .
$$

The residual energy remained on the sensor, after the $t$ th sensing and reporting round, is hence equal to

$$
E_{t, i}=E_{t-1, i}-E_{m s, i}-E_{r p, i}, \quad \text { for } t=1,2, \cdots
$$

\subsection{Proposed Workload Allocation Scheme}

An examination of the energy consumption profile reveals that energy consumed in a sensor depends both on the time used in sensing and the distance between the sensor and the cluster head. However, the distance is fixed once the sensor is deployed and cannot be altered. On the contrary, the time used in sensing and result reporting can be properly determined in 
order to reduce energy usage. The philosophy adopted is that a lighter workload would be assigned to a sensor which a lesser amount of residual energy has remained onboard.

Recall the time $T_{S_{i}}$ that a sensor used for measuring and result reporting, as given by (2.3) and repeated here,

$$
T_{S_{i}}=\alpha_{i} y_{i} T_{m s}+\alpha_{i} z_{i} T_{r p}
$$

Without loss of generality, we make the timing scale factors equal, $y_{i}=z_{i}$. Furthermore, by making use of the fact that the amount of data reported by a sensor is proportional to its sensing time, $T_{m s}=k T_{r p}$, then

$$
T_{S_{i}}=\alpha_{i} y_{i}(1+k) T_{r p}
$$

Now, put the coefficient $y_{i}$ as a function of the ratio of the initial energy $E_{0, i}$ to the instantaneous onboard energy $E_{t, i}$, that is

$$
y_{i}=\frac{E_{0, i}}{E_{t, i}+\epsilon}
$$

where $\epsilon$ is a small positive constant to prevent division by zero.

Consider the recursive equations in calculating the workload allocation $\alpha_{i}$, (2.5), substitute the above assumptions and the energy dependence, we have

$$
\begin{aligned}
s_{i} & =\frac{y_{i-1} k T_{r p}}{y_{i} k T_{r p}+y_{i} T_{r p}} \\
& =\frac{k y_{i-1}}{(1+k) y_{i}} \\
& \approx \frac{k E_{0, i} / E_{t, i-1}}{(1+k) E_{0, i} / E_{t, i}} \\
& \approx \frac{k E_{t, i}}{(1+k) E_{t, i-1}} .
\end{aligned}
$$

Furthermore, the workload ratio for two consecutive sensors is

$$
\alpha_{i}=s_{i} \alpha_{i-1}
$$

and substitute (3.8), then

$$
\frac{\alpha_{i}}{\alpha_{i-1}}=\frac{k E_{t, i}}{(1+k) E_{t, i-1}} .
$$

It can be observed that workloads assigned are now made proportional to the residual energy remained onboard the sensor together with the constant system dependent scaling 
Table 1: Simulation parameters.

\begin{tabular}{lc}
\hline Parameter description & Value \\
\hline Number of simulation runs for each SDLT and EDDLT case & 100 \\
Area of square environment monitored & $50 \times 50 \mathrm{~m}$ \\
Number of sensors $(N)$ & 30 \\
Data required by master processor $(B)$ & $2 \times 10^{6}$ bytes \\
Time to transmit 1 bit $\left(\tau_{b}\right)$ & $64 \times 10^{-6} \mathrm{sec}$ \\
Ratio of sensing/reporting durations $(k)$ & 8 \\
Average battery voltage $(V)$ & $3 \mathrm{~V}$ \\
Battery capacity $(A)$ & $0.5 \mathrm{Ah}$ \\
Current drain per bit $(I)$ & $0.3 \times 10^{-3} \mathrm{~A} / \mathrm{bit}$ \\
\hline
\end{tabular}

factor $k /(1+k)$. Hence, sensors with a lesser amount of residual energy will receive a lesser amount of workload consequently reduces its energy consumption and prolongs its operation lifetime. Furthermore, when the factor $k$ is large, the workload becomes largely dependent on the residual energies.

\section{Simulation}

Simulations are conducted to verify the effectiveness of the proposed energy dependent divisible load theory when it is applied in the workload allocation of a wireless sensor network. Two test cases are studied including the standard DLT (SDLT) approach and the energy dependent DLT (EDDLT) approach. It is assumed that sensors are deployed randomly over an area that is to be monitored. Because of the randomness in initial sensor deployments, the effectiveness will be assessed by a large number of repetitive tests. Statistics are collected on the sensing rounds that the first sensor energy depletes, the sensing rounds that the last sensor depleted its energy, and distribution of residual energies when the first sensor depletes its energy. The simulations conducted are based on the system and sensor parameters given in Table 1.

\subsection{History of Energy Depletion}

In the simulations for SDLT and EDDLT approaches, sensors are deployed randomly over a square sensing area as shown in Figures 3(a) and 4(a), respectively, at the first sensing round. In the figures, a red square is used to represent the cluster head. Sensors are indicated as black dots while their initial energies are denoted by circles whose diameters are proportional to the onboard energy. The instances that the first sensor depletes its energy for the SDLT and EDDLT cases are depicted in Figures 3(b) and 4(b). Since the sensors are deployed randomly and their positions relative to the cluster head would affect the energy consumption, numerical values given here are regarded as typical sample values only.

It can be seen that in the SDLT approach, at a small number of sensing rounds at 1900, other sensors still maintain relative large amount of remaining energies. On the other hand, in the EDDLT case, when the first sensor depletes its energy, energies on other sensors are also depleted to a large extend. In addition, the sensing round at 6244 that the first sensor depletes its energy is much more than that in the SDLT case. Figures 3(c) and 4(c) illustrate the instance 


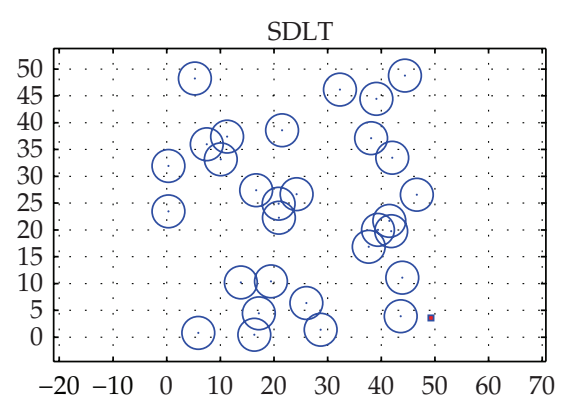

(a)

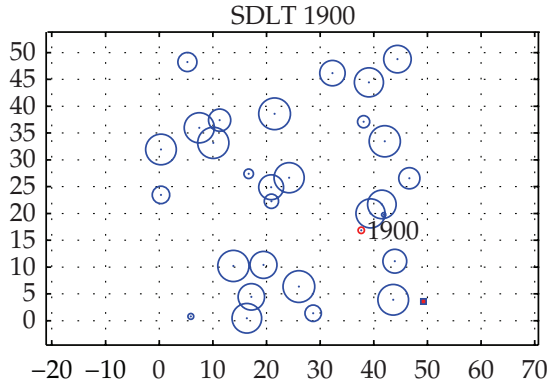

(b)

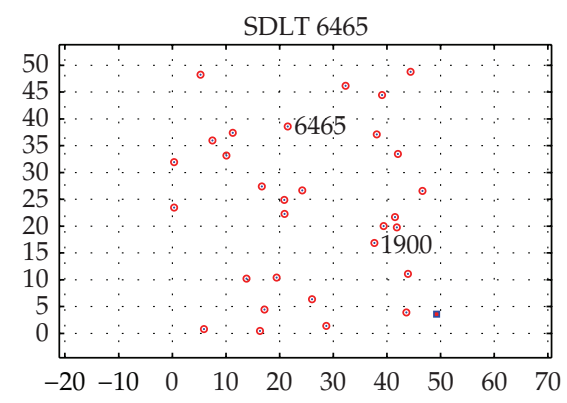

(c)

Figure 3: Sensor energy evolution in standard DLT (SDLT) workload allocation. (a) initial energy onboard sensors, (b) instance when first sensor depleted, (c) instance when last sensor depleted.

when the last sensor depletes its energy. The sensing round conducted in the SDLT case is 6465 while that in the EDDLT case is 6469 . These sensing rounds are approximately the same as expected. It is because the total amount energies inherent in the system and the amount of resultant sensed data are the same.

\subsection{History of Life Sensors in Operation}

Graphical plots depicting the histories of the number of life sensors remaining in operation, for the SDLT and EDDLT cases, are given in Figures 5(a) and 5(b). It can be observed that the first sensor depletes much earlier in the SDLT case as compared with the EDDLT case. Furthermore, the figures also illustrate that the overall system failure times are approximately the same in these two cases. Contradictorily, the slope of sensor death in the EDDLT case is much steeper than the SDLT case. This observation reflects the fact that the EDDLT approach is effective in prolonging the overall operation of the wireless sensor network.

\subsection{Distribution of Energy Onboard Sensors}

The plots of the distribution of energies onboard sensors, when the first sensor depletes, are shown in Figures 6(a) and 6(b). The graph of energy onboard sensors in the SDLT case shows that many sensors still carry substantial amount of energies when the first sensor depletes. This observation indicates that the SDLT strategy for workload allocation is not effective to 


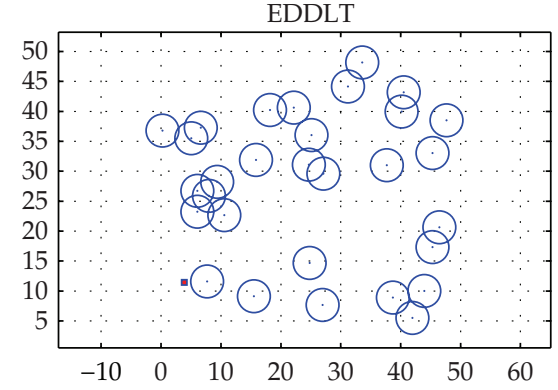

(a)

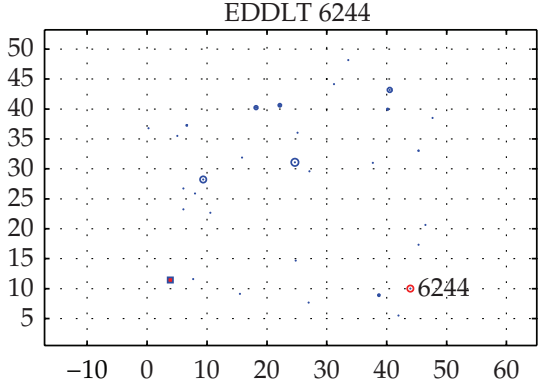

(b)

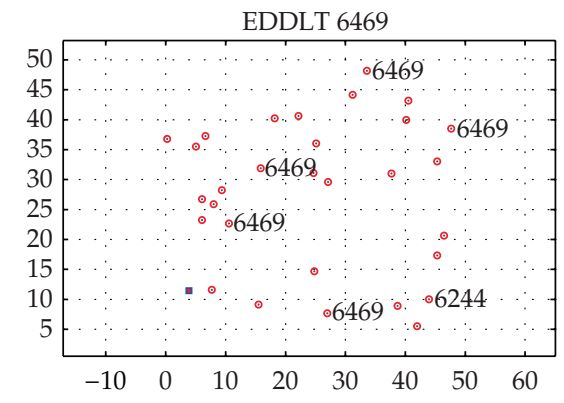

(c)

Figure 4: Sensor energy evolution in energy dependent DLT (EDDLT) workload allocation. (a) initial energy onboard sensors, (b) instance when first sensor depleted, (c) instance when last sensor depleted.

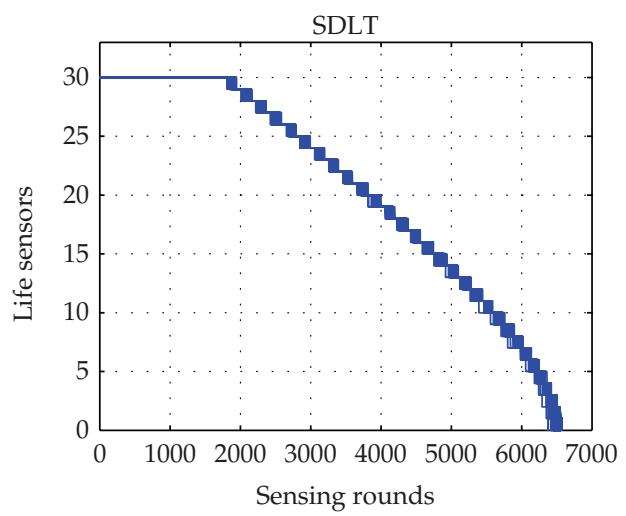

(a)

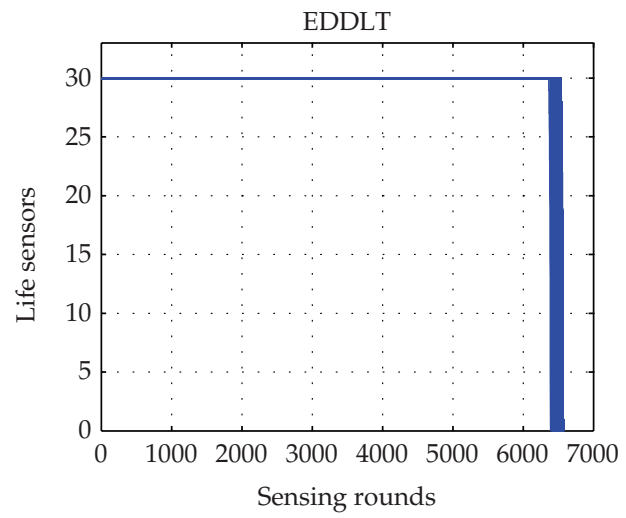

(b)

Figure 5: History of number of life sensors. (a) SDLT case, (b) EDDLT case.

maintain the whole WSN to operation at full capacity. On the other hand, in the EDDLT case, most sensors have used up their energies at later sensing rounds when the first sensor depletes. This reflects the fact that in a large portion of the sensing period, most sensors are in operation and a better monitoring of the environment is made. 


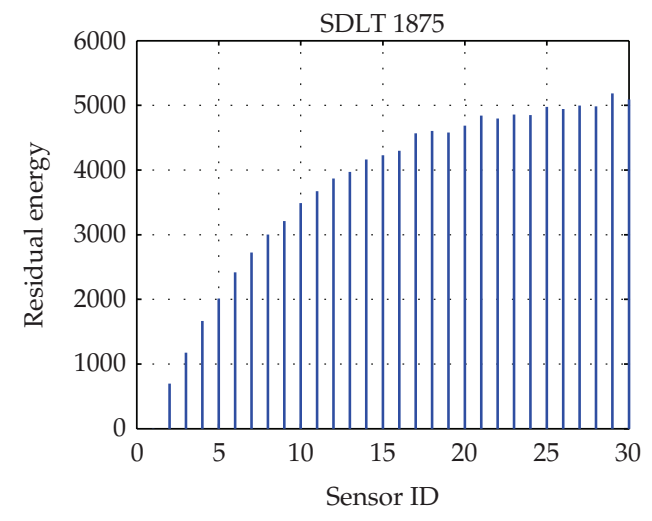

(a)

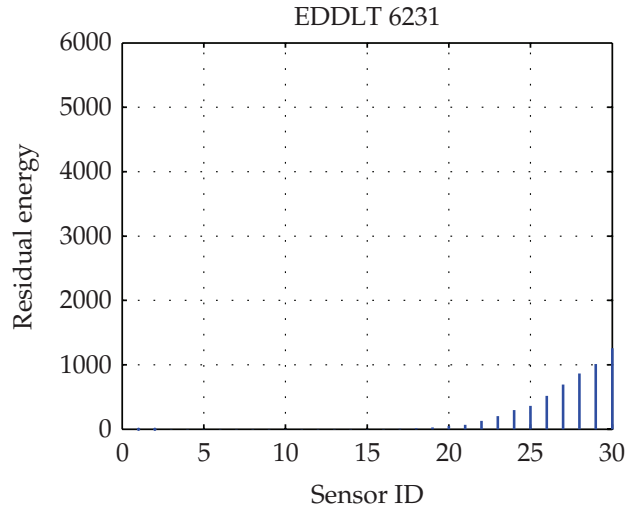

(b)

Figure 6: Distribution of energy onboard sensors. (a) SDLT case, (b) EDDLT case.

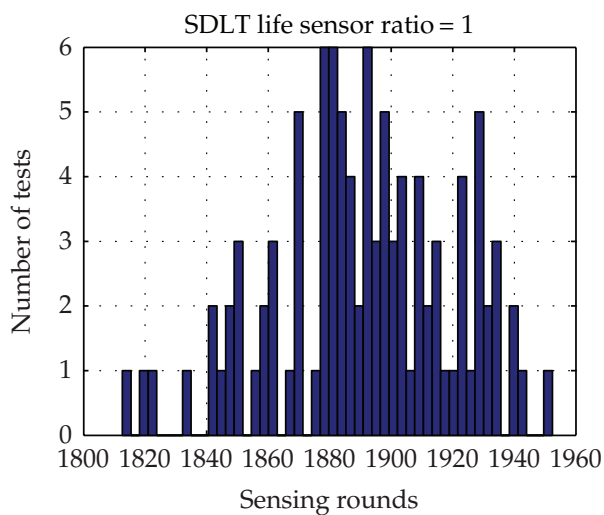

(a)

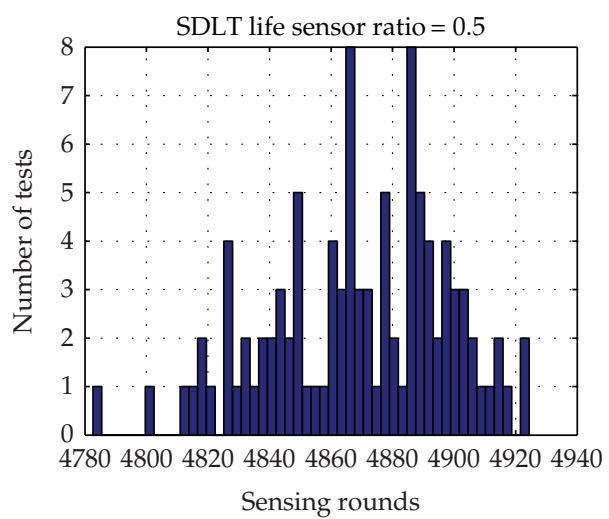

(b)

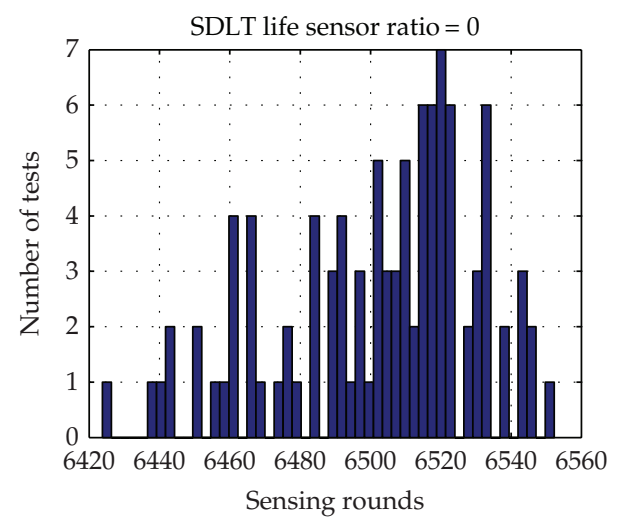

(c)

Figure 7: Statistics of test runs - SDLT case. (a) first sensor depletes, (b) 50\% sensors remain life, (c) last sensor depletes. 


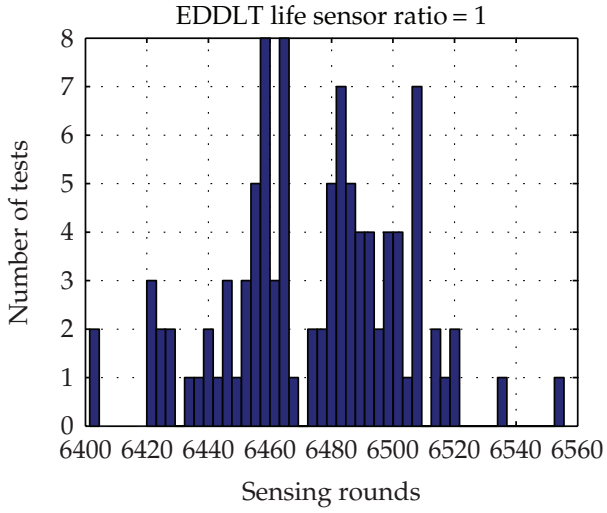

(a)

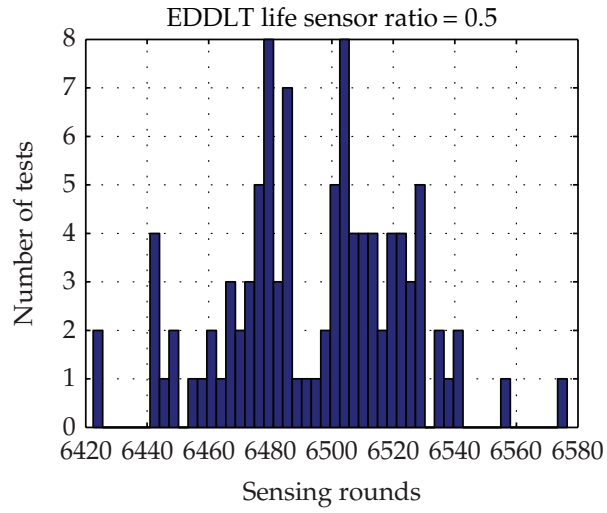

(b)

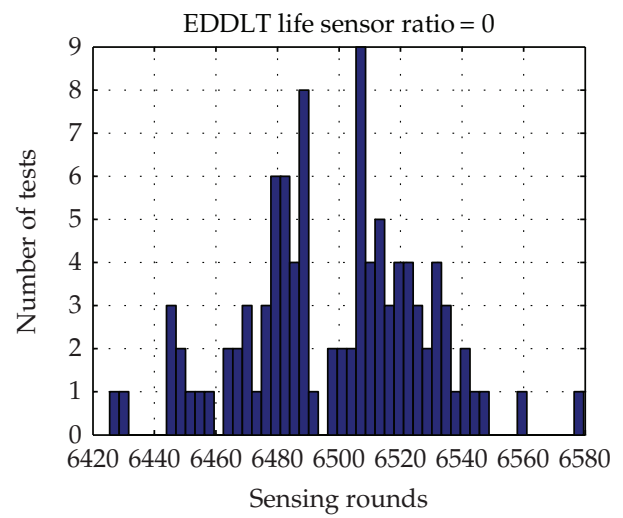

(c)

Figure 8: Statistics of test runs - EDDLT case. (a) first sensor depletes, (b) 50\% sensors remain life, (c) last sensor depletes.

\subsection{Network Performance Statistics}

Since the initial random deployment of sensors critically affects the network performance, its characterization is here based on the statistics from a repetition of 100 simulations. Figures 7 and 8 show, from simulation results from the SDLT and EDDLT cases, the distribution of first sensor depletion, the instance of 50\% sensors remain life in the sensing round when all sensors consumed their energies. The distributions demonstrate that the number of sensing rounds approximately obeys normal distributions in both the SDLT and EDDLT cases, thus, providing statistical evidences on the validity of the results. As it is suggested from other performance measures presented above, the sensing rounds that the first sensor depletes in the EDDLT cases are much later than that of the SDLT cases. Figure 9 further compares the statistics of the ratio of the number of sensors remaining life throughout the sensing task duration. The SDLT case is given in dotted lines with the mean value and $\pm 3 \sigma$ deviations from the mean. It is seen that the EDDLT approach has enabled the network to operate with the full number of sensors at sensing rounds much later than the SDLT case. 


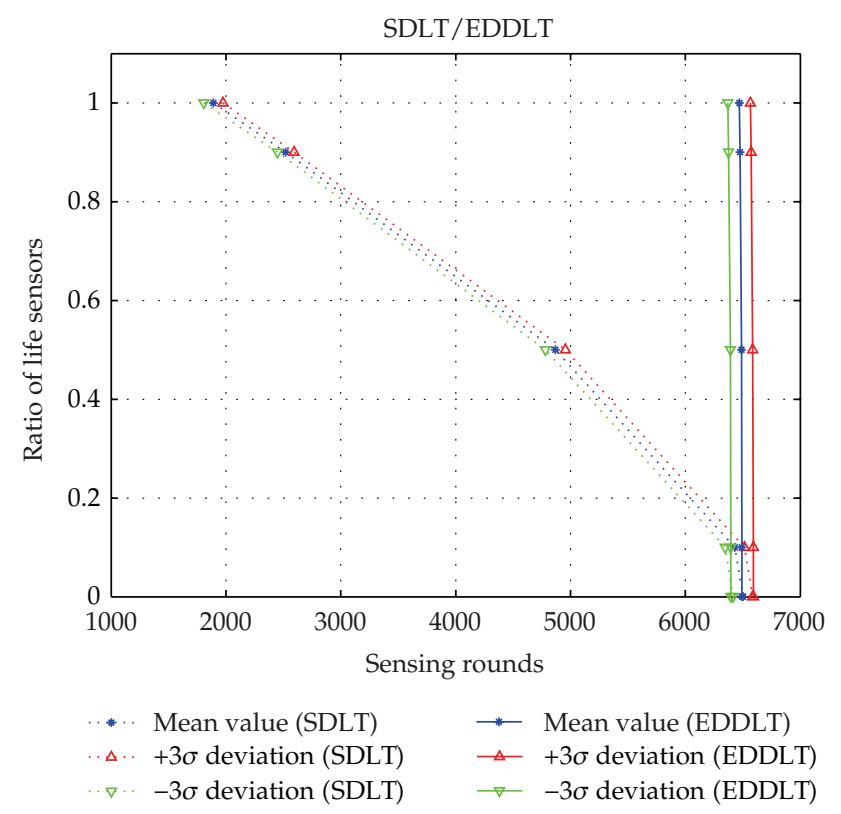

Figure 9: Comparison of ratio of life sensors between standard and energy dependent DLT for workload allocation.

\section{Conclusion}

This paper has presented a study on the wireless sensor network workload allocation scheme. An energy dependent divisible load theory (EDDLT) based approach is developed. In the proposed approach, the residual energies of sensors are considered while assigning the workload to each sensor. Sensors with smaller residual energy are assigned lighter workload. With this strategy, the number of sensing rounds that the first sensor depletes is increased as compared to the standard divisible load theory approach. Experiments are included to illustrate the effectiveness of the EDDLT method in extending the lifespan of the wireless sensor network.

\section{Acknowledgments}

This work was supported by the National Natural Science Foundation of China (Grant nos. 61070043, 60573123), Zhejiang Provincial Key Disciplines on Information Processing \& Automation Technology (20110815), and Zhejiang Education Department (Y201122434).

\section{References}

[1] C. Cattani, S. Chen, and G. Aldashev, "Information and modeling in complexity," Mathematical Problems in Engineering, vol. 2012, Article ID 868413, 3 pages, 2012.

[2] S. Y. Chen, H. Tong, Z. Wang, S. Liu, M. Li, and B. Zhang, "Improved generalized belief propagation for vision processing," Mathematical Problems in Engineering, vol. 2012, Article ID 416963, 12 pages, 2011. 
[3] S. Wen, W. Zheng, J. Zhu, X. Li, and S. Chen, "Elman fuzzy adaptive control for obstacle avoidance of mobile robots using hybrid force/position incorporation," IEEE Transactions on Systems, Man and Cybernetics C, vol. 42, no. 4, pp. 603-608, 2012.

[4] J. Yick, B. Mukherjee, and D. Ghosal, "Wireless sensor network survey,” Computer Networks, vol. 52, no. 12, pp. 2292-2330, 2008.

[5] H. Y. Shi, W. L. Wang, N. M. Kwok, and S. Y. Chen, "Game theory for wireless sensor networks: a survey," Sensors, vol. 12, no. 7, pp. 9055-9097, 2012.

[6] K. Akkaya and M. Younis, "A survey on routing protocols for wireless sensor networks," Ad Hoc Networks, vol. 3, no. 3, pp. 325-349, 2005.

[7] G. Anastasi, M. Conti, M. Di Francesco, and A. Passarella, “Energy conservation in wireless sensor networks: a survey," Ad Hoc Networks, vol. 7, no. 3, pp. 537-568, 2009.

[8] A. Kansal and M. B. Srivastava, "An Environmental Energy Harvesting Framework for Sensor Networks," in Proceedings of the International Symposium on Low Power Electronics and Design (ISLPED '03), pp. 481-486, August 2003.

[9] C. K. Toh, "Maximum battery life routing to support ubiquitous mobile computing in wireless ad hoc networks," IEEE Communications Magazine, vol. 39, no. 6, pp. 138-147, 2001.

[10] H. Y. Shi, N. M. Kwok, and W. L. Wang, "Mechanism penalty for task scheduling in wireless sensor networks," Advances in Information Sciences and Service Sciences, vol. 4, no. 11, pp. 233-242, 2012.

[11] T. G. Robertazzi, “Ten reasons to use divisible load theory,” Computer, vol. 36, no. 5, pp. 63-68, 2003.

[12] J. Błażewicz, M. Drozdowski, and M. Markiewicz, "Divisible task scheduling—concept and verification," Parallel Computing, vol. 25, no. 1, pp. 87-98, 1999.

[13] V. Bharadwaj, D. Ghose, and T. G. Robertazzi, "Divisible load theory: a new paradigm for load scheduling in distributed systems," Cluster Computing, vol. 6, pp. 7-17, 2003.

[14] J. Sohn and T. G. Robertazzi, "Optimal time-varying load sharing for divisible loads," IEEE Transactions on Aerospace and Electronic Systems, vol. 34, no. 3, pp. 907-923, 1998.

[15] J. Sohn, T. G. Robertazzi, and S. Luryi, "Optimizing computing costs using divisible load analysis," IEEE Transactions on Parallel and Distributed Systems, vol. 9, no. 3, pp. 225-234, 1998.

[16] O. Beaumont, H. Casanova, A. Legrand, Y. Robert, and Y. Yang, "Scheduling divisible loads on star and tree networks: results and open problems," IEEE Transactions on Parallel and Distributed Systems, vol. 16, no. 3, pp. 207-218, 2005.

[17] C. F. Gamboa and T. G. Robertazzi, "Efficient scheduling for sensing and data reporting in wireless sensor networks," in Proceedings of the 40th Annual Conference on Information Sciences and Systems (CISS '06), pp. 171-176, March 2006.

[18] M. Moges and T. G. Robertazzi, "Wireless sensor networks: scheduling for measurement and data reporting," IEEE Transactions on Aerospace and Electronic Systems, vol. 42, no. 1, pp. 327-340, 2006.

[19] M. Drozdowski, "Energy considerations for divisible load processing," in Proceedings of the 8th International Conference on Parallel Processing and Applied Mathematics (PPAM '09), Lecture Notes in Computer Science, pp. 92-101, 2009.

[20] W. R. Heinzelman, A. Chandrakasan, and H. Balakrishnan, "Energy-efficient communication protocol for wireless microsensor networks," in Proceedings of the 33rd Annual Hawaii International Conference on System Siences (HICSS '00), p. 223, January 2000.

[21] J. S. Lee and W. L. Cheng, "Fuzzy-logic-based clustering approach for wireless sensor networks using energy prediction," IEEE Sensors Journal, vol. 12, no. 9, pp. 2891-2896, 2012.

[22] J. Xue, M. Li, W. Zhao, and S. Chen, "Bound maxima as a traffic feature under DDOS flood attacks," Mathematical Problems in Engineering, vol. 2012, Article ID 419319, 20 pages, 2012.

[23] S. Castellucci and M. Carlini, "Modelling and simulation for energy production parametric dependence in greenhouses," Mathematical Problems in Engineering, vol. 2010, Article ID 590943, 28 pages, 2010.

[24] S. C. Lim, C. H. Eab, K. H. Mak, M. Li, and S. Y. Chen, "Solving linear coupled fractional differential equations by direct operational method and some applications," Mathematical Problems in Engineering, vol. 2012, Article ID 653939, 28 pages, 2012.

[25] S. Chen, W. Huang, C. Cattani, and G. Altieri, "Traffic dynamics on complex networks: a survey," Mathematical Problems in Engineering, vol. 2012, Article ID 732698, 23 pages, 2012.

[26] M. Li, S. C. Lim, and S. Chen, "Exact solution of impulse response to a class of fractional oscillators and its stability," Mathematical Problems in Engineering, vol. 2011, Article ID 657839, 9 pages, 2011.

[27] S. Castellucci, M. Carlini, M. Guerrieri, and T. Honorati, "Stability and control for energy production parametric dependence," Mathematical Problems in Engineering, vol. 2010, Article ID 842380, 21 pages, 2010. 
[28] W. B. Heinzelman, A. P. Chandrakasan, and H. Balakrishnan, "An application-specific protocol architecture for wireless microsensor networks," IEEE Transactions on Wireless Communications, vol. 1, no. 4, pp. 660-670, 2002.

[29] M. Younis, M. Youssef, and K. Arisha, "Energy-aware management for cluster-based sensor networks," Computer Networks, vol. 43, no. 5, pp. 649-668, 2003.

[30] S. Fedor and M. Collier, "On the problem of energy efficiency of multi-hop vs one-hop routing in Wireless Sensor Networks," in Proceedings of the 21st International Conference on Advanced Information Networking and ApplicationsWorkshops/Symposia (AINAW'07), pp. 380-385, May 2007.

[31] S. Hussain and O. Islam, "An energy efficient spanning tree based multi-hop routing in wireless sensor networks," in Proceedings of IEEE Wireless Communications and Networking Conference (WCNC '07), pp. 4386-4391, March 2007.

[32] J. Yu, Y. Qi, G. Wang, Q. Guo, and X. Gu, "An energy-aware distributed unequal clustering protocol for wireless sensor networks," International Journal of Distributed Sensor Networks, vol. 2011, Article ID 202145, 8 pages, 2011.

[33] X. L. Li and J. N. Cao, "Coordinated workload scheduling in hierarchical sensor networks for data fusion applications," Journal of Computer Science and Technology, vol. 23, no. 3, pp. 355-364, 2008.

[34] D. Ghose, H. J. Kim, and T. H. Kim, "Adaptive divisible load scheduling strategies for workstation clusters with unknown network resources," IEEE Transactions on Parallel and Distributed Systems, vol. 16, no. 10, pp. 897-907, 2005.

[35] R. Min, M. Bhardwaj, S. H. Cho et al., "Architecture for a power-aware distributed microsensor node," in Proceedings of the Workshop on Signal Processing Systems (SIPS '00), pp. 581-590, October 2000.

[36] O. Younis and S. Fahmy, "An experimental study of routing and data aggregation in sensor networks," in Proceedings of the 2nd IEEE International Conference on Mobile Ad-hoc and Sensor Systems (MASS '05), pp. 50-57, November 2005.

[37] C. H. Ou, "A localization scheme for wireless sensor networks using mobile anchors with directional antennas," IEEE Sensors Journal, vol. 11, no. 7, pp. 1607-1616, 2011.

[38] G. Hou and K. W. Tang, "Evaluation of LEACH protocol subject to different traffic models," in Proceedings of the 1st International Conference on Next Generation Network, pp. 281-283, 2006. 


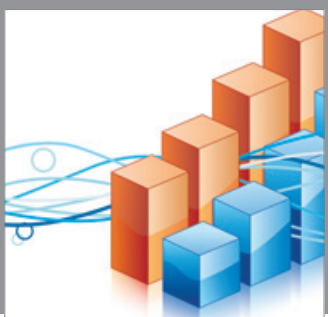

Advances in

Operations Research

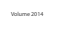

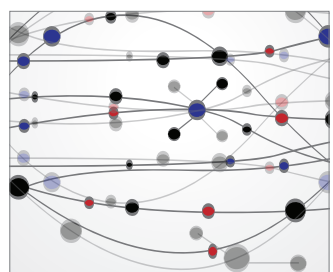

\section{The Scientific} World Journal
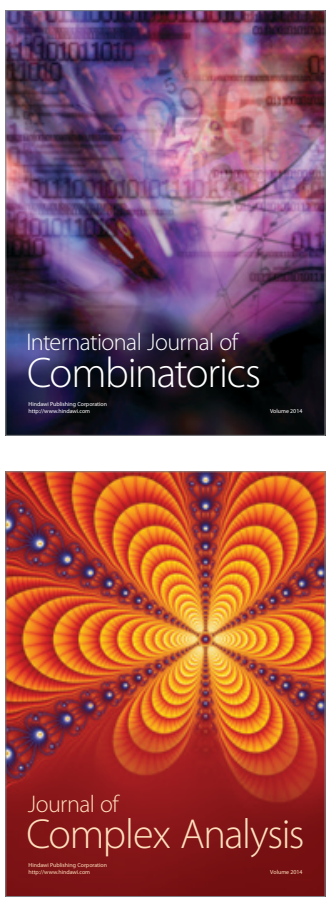

International Journal of

Mathematics and

Mathematical

Sciences
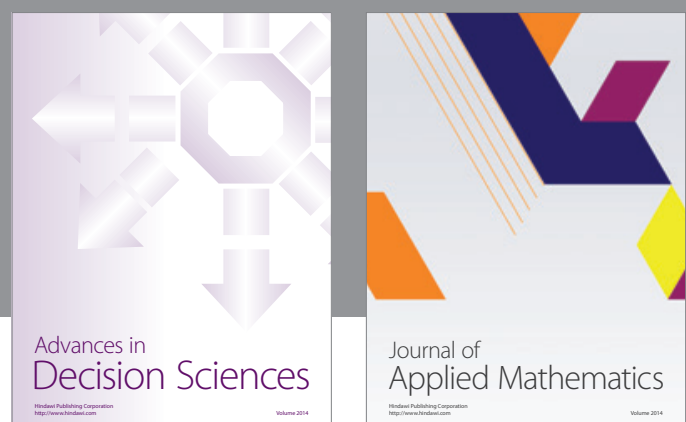

Journal of

Applied Mathematics
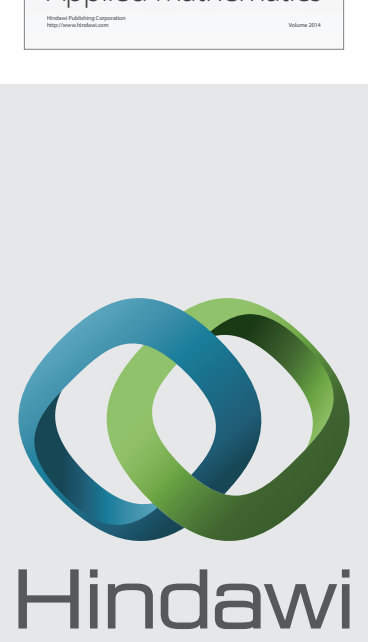

Submit your manuscripts at http://www.hindawi.com
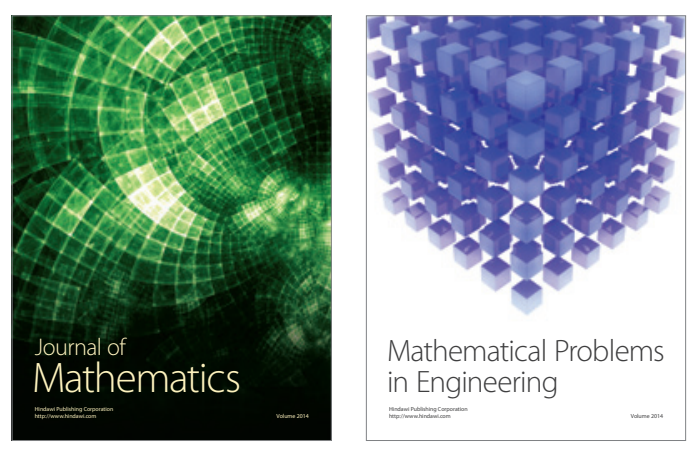

Mathematical Problems in Engineering
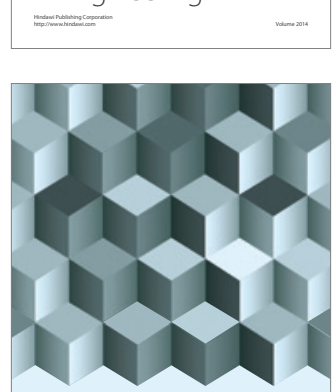

Journal of

Function Spaces
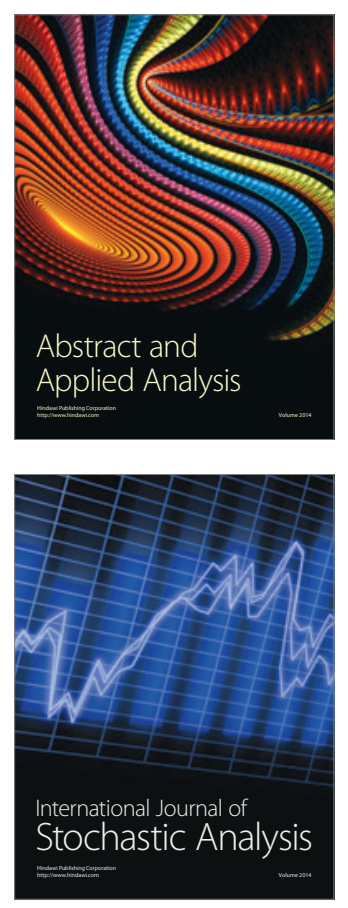

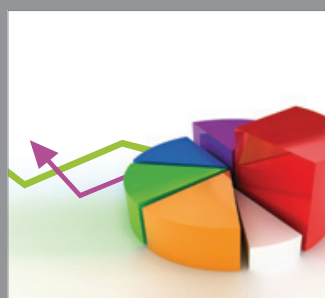

ournal of

Probability and Statistics

Promensencen
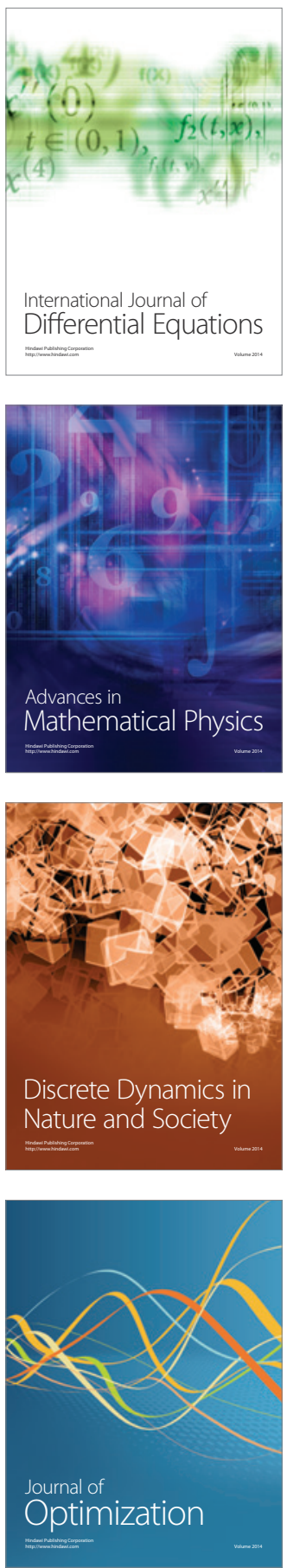